\section{Visual cortical evoked potentials under conditions of sequential blanking*}

\section{J. L. ANDREASSI, M. S. MAYZNER, D. R. BEYDA, and S. DAVIDOVICS New York University, Bronx, New York 10453}

Two separate experiments were conducted to study the visual evoked potential (VEP) correlates of sequential blanking, a phenomenon in which up to one-half of a discrete train of stimulus inputs are not perceived for certain orders of input. A PDP-7 digital computer was used to present stimuli (from 2 to 5 letter Xs) on a CRT display in both experiments. In Experiment 1 there were four basic conditions designed and counterbalanced to indicate the nature of the VEP when stimuli were blanked and when Ss reported all stimuli. All of the stimuli were of equal intensity. The main finding was that although the eight Ss did not perceive and report blanked stimuli, they did respond to them physiologically as indicated by the VEP. The implications of these findings were discussed in relation to recent studies of visual masking and metacontrast in which VEP was recorded. In Experiment 2 the normally blanked and the normally blanking stimuli were alternately increased in intensity to determine the effect on sequential blanking and the VEP. Six Ss were tested under three basic counterbalanced conditions. It was found that sequential blanking could be reliably overcome by increasing the intensity of the normally blanked stimuli. In addition, when the normally blanking stimuli were of greater intensity than the blanked stimuli, not only did perceptual suppression occur, but the evideisce indicated that there was no VEP to the first of the two blanked stimuli.

The phenomenon of sequential blanking has been thoroughly described by Mayzner, Tresselt, and Helfer (1967) and further elaborated upon by Mayzner and Tresselt (1970). Briefly, sequential blanking refers to the finding that if a discrete train of stimuli, occurring in different spatial locations on the display surface of a CRT console, are presented sequentially at certain critical input rates, approximately one-half of these stimuli are not perceived. The object of the present experiment was to determine the nature of visual evoked potentials (VEPs) under conditions in which Ss saw all of the stimuli and when some stimuli were blanked.

There have been several studies which have explored the VEP correlates of phenomena such as metacontrast and visual masking (Schiller \& Chorover, 1966; Vaughan \& Silverstein, 1968; Donchin, Wicke, \& Lindsley, 1963; Donchin \& Lindsley, 1965). Schiller and Chorover (1966) investigated the question of whether or not brightness reduction observed under metacontrast conditions (where brightness changes but intensity does not) is correlated with changes in the VEP. They reported no changes in the VEP associated with the reduction of brightness induced by metacontrast and concluded that the VEP does not necessarily reflect changes in

*This research was supported by the Physiological Psychology Program, Office of Naval Research, under ONR Contract No. N00014-67-A-0467-0009 and ONR Contract Authority No. NR 140-252 to the first two authors. subjective brightness. Vaughan and Silverstein (1968) report attenuation of VEPs to foveal stimulation during metacontrast suppression and conclude that the reason for the failure of VEPs to reflect metacontrast suppression in the Schiller and Chorover study was because parafoveal stimulation may have given rise to VEPs generated largely by stray light impinging on the fovea. The attenuation occurred to the VEP component with a maximum amplitude which occurred about $200 \mathrm{msec}$ after the first stimulus. Donchin, Wicke, and Lindsley (1963) studied VEPs under the visual masking paradigm in which the second, brighter flash (BF) masks perception of the initial test flash (TF), at interstimulus intervals (ISI) of 0 to $25 \mathrm{msec}$. At the 20-msec ISI, when visual masking occurred, VEPs were like those of the $B F$ at the $500-m s e c$ ISI, or when presented alone. A similar result obtained by Donchin and Lindsley (1965) led to the interpretation that the interference of $\mathrm{TF}$ by $\mathrm{BF}$ takes place at or preceding the point at which VEPs are recorded (occipital cortex). They expressed the opinion that the same processes which are involved in perceptual blanking seem to be involved in the blocking of the VEP. It will be our purpose in the present investigation to explore the nature of VEPs under sequential blanking conditions in which all of the stimuli are of equal intensity.

\section{EXPERIMENT 1}

Method

Subjects. The Ss were eight male undergraduate, graduate, and faculty members of the School of Engineering and Science at New York University. None had visual defects other than myopia (corrected to $20 / 20$ ).

Apparatus and procedure. Ss were seated in an electrically shielded sound-attenuated room (IAC chamber). All experimental sessions were conducted with the lights out, with Ss dark adapting for $15 \mathrm{~min}$ at the beginning of each session.

The EEG was recorded from $\mathrm{O}_{2}$ (10-20 system, Jasper, 1958) with a Grass silver cup electrode referenced to a silver clip electrode on S's left ear lobe. Electrode resistance was 5,000 ohms or less. The $\mathbf{S}$ was grounded by means of an electrode attached to his left wrist leading to "patient ground" of an Offner RM dynograph. The $9806 \mathrm{~A}$ coupler of the dynograph was used to condition the EEG signal (bandpass set at 0.5 to $32.0 \mathrm{~Hz}$ ). The filtered and amplified signal was fed into a Mnemotron Computer of Average Transients (CAT 1000). A "start" signal triggered the CAT to take EEG samples every $0.5 \mathrm{msec}$ for a $500-\mathrm{msec}$ duration following the presentation of each stimulus to $S$. After 150 stimulus presentations for each of the four stimulus configurations, the summated responses from CAT memory were plotted by a Hewlett-Packard X-Y plotter.

The stimuli were displayed to Ss on a 343 CRT display driven by a PDP-7 digital computer. The basic stimulus configuration used to study sequential blanking in this experiment was a single line of five Xs: X X X X X. Each character was displayed sequentially with $O N$ and OFF times of $20 \mathrm{msec}$, i.e., the first $X$ was presented for $20 \mathrm{msec}$ (ON time) followed by a 20-msec pause (OFF time), and the next $X$ was displayed, etc., until all five Xs were presented. The ISI between groups of five $\mathrm{Xs}$ was set at $1.0 \mathrm{sec}$

Instructions to $\mathbf{S}$ emphasized watching the CRT closely, to report the number of Xs seen after each presentation, and to avoid blinking of the eyes during stimulus presentations. Past research (e.g., Mayzner, 1968) and pilot work for the present experiment indicated that if characters are displayed from left to right (display order of $1,2,3,4,5$ ) Ss report five Xs. A display order of $3,1,4,2,5$, i.e., the $\mathrm{X}$ in the second position from the left appears first, the $X$ in the fourth position second, etc., results in sequential blanking, i.e., $\mathrm{S}$ does not see the second and fourth $X s$ in the line of five Xs. It should be noted that the two display orders selected (i.e., 12345 and 31425) for comparing blanking vs no blanking effects are 


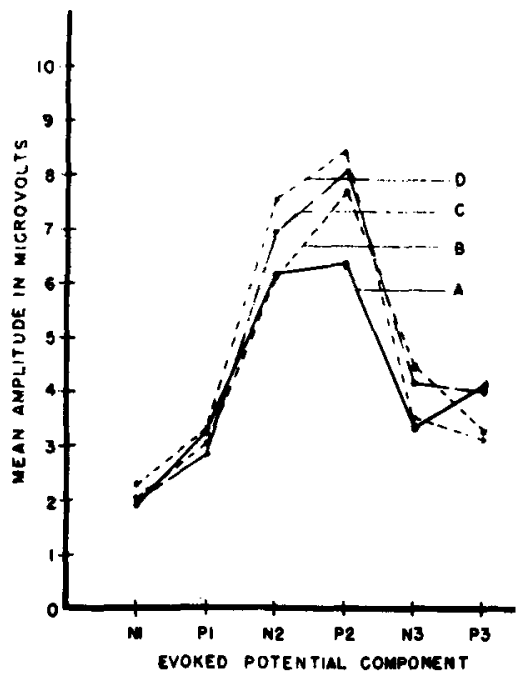

Fig. 1. Mean amplitude (eight Ss) of VEP components under Conditions $A$ (no blanking), B (blanking), C (two $\mathrm{Xs}$ ), and $\mathrm{D}$ (three $\mathrm{Xs}$ ).

only two orders out of a possible total of 120 display orders obtainable with five elements (i.e., 5!). Further experiments are contemplated to explore a wide range of spatiotemporal input display orders and their effects on associated VEPs.

The four stimulus conditions used are shown in Table 1 .

Conditions $\mathrm{A}$ and $\mathrm{B}$ were used to compare no blanking vs blanking effects. Conditions $C$ and $D$ were used as controls.

Each experimental session consisted of four practice trials and eight experimental runs of 150 presentations each. The $\mathrm{Ss}$ rested 1 min between runs. Stimulus conditions were counterbalanced among Ss by a Latin-square design. Each $\mathbf{S}$ was tested on 3 different days, at approximately the same time, resulting in six VEP traces for each condition.

The $\mathrm{S}$ viewed the CRT display from inside the IAC chamber. At a distance of 45 in., the vertical dimension of a single $\mathrm{X}$ produced a visual angle of $39 \mathrm{~min}$ at the eye, the horizontal dimension resulted in a visual angle of $24 \mathrm{~min}$, while a row of five $\mathrm{Xs}$ produced a visual angle of $2 \mathrm{deg}$ $40 \mathrm{~min} .1$ The intensity of each character was the same, $.106 \mathrm{~mL}$, as measured by a Gamma Scientific photometer from inside the IAC chamber, for each steady-state $\mathrm{X}$. Characters with display order numbers
$1,2,3,4$, and 5 always appeared at times $0,40,80,120$, and $160 \mathrm{msec}$, respectively. The CAT was always triggered at time $0 \mathrm{msec}$.

\section{Results and Discussion}

The mean amplitudes (microvolts) and latencies (milliseconds) were obtained for the major positive and negative VEP components from the $\mathrm{X}-\mathrm{Y}$ tracings. The amplitude of the $\mathrm{N} 1$ component was measured as the vertical distance from baseline to the trough of the first depression to occur in the tracing. The P1 component was measured as the vertical distance from $\mathrm{N} 1$ to the peak of the first positive component, while $\mathrm{N} 2$ was measured from P1 to the trough of the second major depression, etc., for P2, N3, and P3. Latencies (or time after stimulus presentation) were measured to the midpoints of each positive and negative component. The amplitude differences of $\mathrm{P} 2$, the largest positive VEP component, under each condition were tested by $t$ tests for correlated data. A two-tailed criterion for significance was used throughout. The tests showed that Condition A produced Iower amplitude P2 components when compared to Conditions $B(t=5.13), C(t=5.27)$, and $D(t=6.21)(p<.01$, df $=7$, for each). Figure 1 shows clearly that P2 for Conditions $B, C$, and D is larger than under Condition A. Examination of the mean amplitude data for each $S$ revealed that all eight $S s$ had higher amplitude P2 with Conditions B, C, and $D$ than with Condition $A$, a consistency which is reflected in the significant differences found.

The mean latency data and tests indicate that, taking $\mathrm{P} 2$ as the criterion, Condition D produced longer latency VEPs than A $(\mathrm{t}=25.88), \quad \mathrm{B} \quad(\mathrm{t}=21.70)$, or $\mathrm{C}$ $(t=25.80)(p<.01, d f=7$, for each $)$. This result is expected, since the first $X$ in Condition $D$ appears $80 \mathrm{msec}$ later than the first $X$ of the other conditions. Figure 2 shows the plot of the latency data.

The VEP tracings for each of the eight Ss were superimposed for each of the conditions. A marked consistency was observed for each condition within the same $S$, even though experimental sessions were conducted over a several-week period. Under Condition A, Ss consistently reported seeing five $\mathrm{Xs}$ which appeared to move from left to right. In $B$ they reported

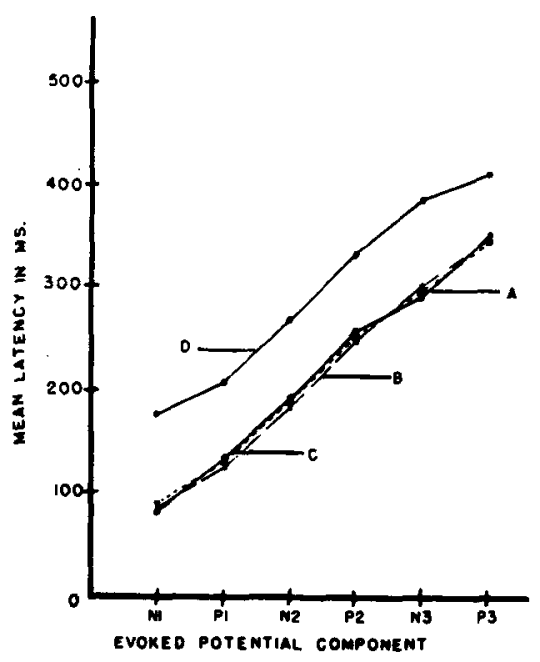

Fig. 2. Mean latencies (eight Ss) for VEP components under Conditions $A$ (no blanking), B (blanking), C (two $X s$ ), and D (three Xs).

only three $\mathrm{Xs}$ consistently, while in $\mathrm{C}$ they reported two, and for Condition $D$ three $X s$ were consistently reported. Figure 3 shows the superimposed VEP tracings for one of the Ss (J.A.), and it can be seen that the traces for Condition $A$ (no blanking) and $B$ (blanking) are different in amplitude. However, the waveforms for Condition B (blanking) and $\mathrm{C}$ (two $\mathrm{Xs}$ ) are more similar to each other. The waveform for Condition D (three Xs) is displaced temporally to the right. Similar results were obtained for the remaining seven Ss, i.e., the VEP under Condition $C$ (when only the first two $\mathrm{Xs}$ were presented) was not different with respect to amplitude, latency, and $w$ aveform from the VEP under Condition B when these same two Xs were not reported as being seen. This finding suggests strongly that although Ss do not perceive and report these two blanked stimuli, they do respond to them physiologically, as indicated by the VEP. This finding further suggests that either (1) the recordings obtained were not from the "primary" visual cortex or (2) that there are "higher" cortical areas other than the visual involved in the final processing stages of visual stimuli. The first possibility points to the problem of recording from the striate or primary visual cortex (area 17), which is principally on the mesial surface of the brain, as pointed out by Donchin et al (1963). It is likely that electrodes

Table 1

Table 2

\begin{tabular}{|c|c|c|c|c|c|}
\hline \multirow{3}{*}{ 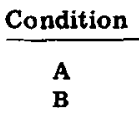 } & \multicolumn{4}{|c|}{ Characters-Location } & \multirow{2}{*}{$\frac{\text { Display Order }}{1,2,3,4,5}$} \\
\hline & $\mathbf{x}$ & $\mathbf{x}$ & $\mathbf{x}$ & $\mathbf{x}$ & \\
\hline & $\mathbf{x}$ & $\mathbf{x}$ & $\mathbf{x}$ & $\mathbf{x}$ & $3,1,4,2,5$ \\
\hline $\mathbf{C}$ & & $\mathbf{x}$ & & $\mathbf{x}$ & 12 \\
\hline $\mathbf{D}$ & $\mathbf{x}$ & & $\mathbf{X}$ & $\mathbf{x}$ & 45 \\
\hline
\end{tabular}

\begin{tabular}{|c|c|c|c|c|c|c|c|c|c|}
\hline \multirow{2}{*}{$\frac{\text { Condition }}{A}$} & \multicolumn{4}{|c|}{ Characters-Location } & \multicolumn{5}{|c|}{ Display Order } \\
\hline & $\mathbf{X}$ & $\underline{\mathbf{x}}$ & $\mathbf{x}$ & $\underline{x} \mathrm{x}$ & $\mathbf{3}$ & 1 & 4 & 2 & $\mathbf{5}$ \\
\hline $\mathbf{B}$ & $\underline{x}$ & $\mathbf{x}$ & $\underline{x}$ & $\mathrm{x} \underline{\mathrm{x}}$ & 3 & $\mathbf{1}$ & 4 & $\mathbf{2}$ & 5 \\
\hline C & & $\mathbf{x}$ & & $x$ & & 1 & & 2 & \\
\hline
\end{tabular}


A
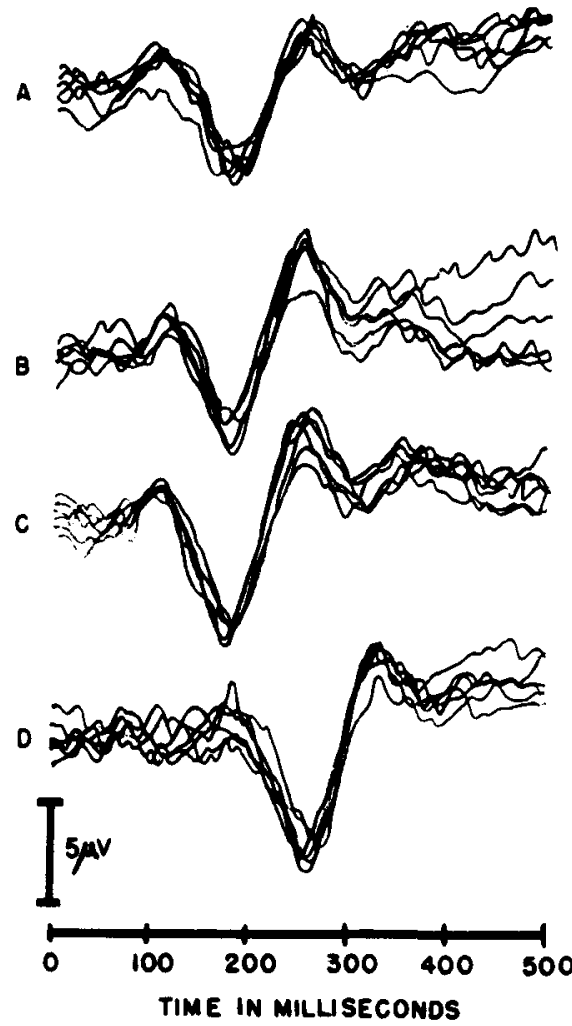

Fig. 3. VEPs for one S (J.A.) under Conditions A (no blanking), B (blanking), C (two Xs), and D (three $\mathrm{Xs)}$. Negativity is downward.

placed on the scalp over the occipital cortex will record from prestriate or "visual association" areas 18 or 19 of Brodman (see Morgan, 1965). The problem revolves around the question of how much more "primary" is area 17 , as compared to areas 18 and 19 , in the kind of perceptual task used in the present experiment, in determining what is seen and what is not seen. This question is not likely to be answered until more is known regarding the functioning of these three areas with respect to the processing of sequentially presented visual stimuli.

The second possibility regarding "higher" cortical areas receives some support from Hubel (1963) who, describing his work with cats, has expressed the view that the visual cortex is only an early stage of the visual pathway and points out that little is known regarding pathways leading from the visual cortex. In any event, the results obtained in the present experiment emphasize the importance of recording from over other areas of the cortex to explore the possibility that areas normally active during no blanking conditions become silent during blanking.

The result indicating that the P2 component was significantly lower under Condition $A$, in which all stimuli were perceived, than under the other three conditions may be due to attentional factors. That is, Condition A has a smooth flowing intact appearance and may have commanded less of S's attention as compared to the broken, and essentially more complex, pattern of Condition B. There is research evidence which indicates that attention to stimuli results in greater amplitude VEPs (e.g., see Donchin \& Cohen, 1967; Eason, Harter, \& White, 1969; and Groves \& Eason, 1969).

The results of the present experiment are not like those of the visual masking experiments reported by Donchin et al (1963) and Donchin and Lindsley (1965), in which VEPs were found to occur in response to the masking (second) rather than the masked (first) stimuli. However, under the visual masking paradigm, the second flash is brighter than the first, while in our study all of the stimuli were of equal intensity. In addition, stimuli presented in the sequential blanking paradigm are in adjacent locations, while they appear in the same location in visual masking.

The results of our study are similar to both those of Schiller and Chorover (1966) and Vaughan and Silverstein (1968) for parafoveal stimuli. It will be recalled that Schiller and Chorover (1966) obtained no reduction of VEP under conditions of metacontrast suppression, while Vaughan and Silverstein (1968) report a reduction in VEP under metacontrast for foveal stimuli, but not for parafoveal stimuli. Therefore, it appears that stimuli which are subjectively blanked or reduced in brightness can produce sizable responses in the visual cortex, as indicated by the VEP.

To return to the question of disparity between the results of the present experiment and those of Donchin et al (1963) and Donchin and Lindsley $(1965)$, it seemed that the next natural step would be to study sequential blanking and associated VEPs under conditions in which the blanked and blanking stimuli were alternately of greater intensity. Experiment 2, therefore, was aimed at investigating the effects of stimulus intensity variations on sequential blanking and the VEP.

\section{EXPERIMENT 2}

The questions asked in conducting this experiment were: (1) Can sequential blanking be overcome by increasing the intensity of the two blanked characters? (2) What occurs when the intensity of the blanking characters is increased relative to that of the blanked ones? (3) What is the nature of the VEP under these conditions?

\section{Method}

Subjects. The Ss were six male undergraduates, graduate students, and faculty members in the School of Engineering and Science at New York University. None of the Ss had visual defects other than corrected myopia (three Ss).

Apparatus. The apparatus used was the same as that used in Experiment 1.

Procedure. There were three basic conditions in this experiment (see Table 2).

In Condition A, a display order which resulted in sequential blanking was used, but the intensities of the two normally blanked Xs (underlined, temporal order 1 and 2) were raised until $S$ always reported five Xs. In effect, sequential blanking was reliably overcome by increasing the intensity of these Xs for each of the six Ss during pilot sessions. In Condition B the intensities of the three normally blanking Xs (underlined, temporal order 3,4 , and 5 ) were raised, and, in effect, sequential blanking was strengthened. In Condition C, two Xs, of the same intensity as those blanked (temporal order 1 and 2 ) in Condition B were used. The intensities were varied by separate potentiometers built into the CRT display. The light intensities produced by the potentiometer settings were calibrated for a single $\mathrm{X}$ with a Gamma Scientific photometer. The photometer was placed inside the darkened IAC chamber and at the normal position of S's eyes (45 in. from the display surface) during calibration.

A pilot study was conducted to determine the intensity required to overcome blanking. It was found that for Condition A blanking could be overcome by increasing the intensities of the two blanked Xs to $.07 \mathrm{~mL}$, as compared to $.02 \mathrm{~mL}$ for the normally blanking Xs. For Condition B the intensities were reversed, i.e., the blanked Xs were at $.02 \mathrm{~mL}$ while the blanking $X s$ were at $.07 \mathrm{~mL}$. In Condition $\mathrm{C}$ the two $\mathrm{Xs}$ were each at $.02 \mathrm{~mL}$

Three VEP tracings were recorded for each condition, on 2 separate days, for each $S$, resulting in six traces for each condition. $\mathrm{S}$ was tested at approximately the same time of day in each session. Each trace was based on 150 stimulus presentations.

\section{Results}

The mean amplitudes and latencies for each of the VEP components for each $S$ were computed in the same manner as in Experiment 1.

Figure 4 clearly shows the higher amplitude of the P2 component for Condition $A$ as compared to $C$ and $B$. It is interesting to note that the 


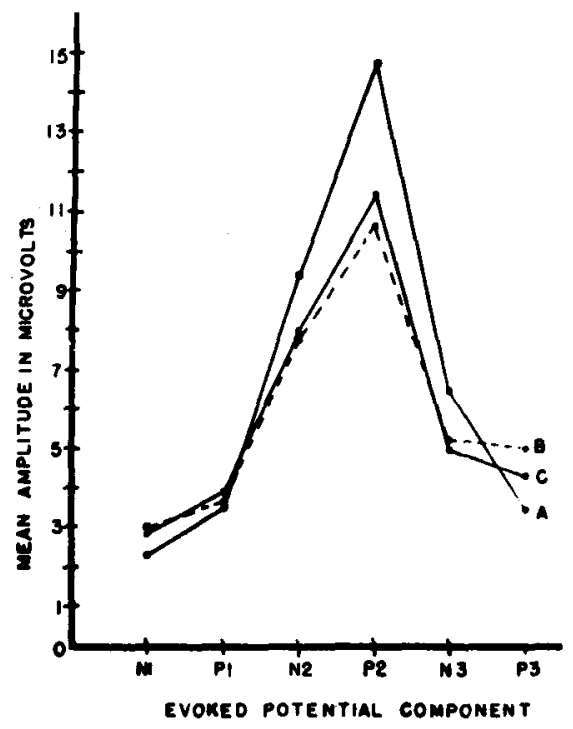

Fig. 4. Mean amplitudes (six $S_{\S}$ ) for VEP components under Conditions $A$ (blanking overcome), B (blanking), and $\mathrm{C}$ (two Xs).

average amplitude of $\mathbf{P 2}$ in Condition $\mathbf{C}$ is slightly higher than in Condition $B$, even though the first two $\mathrm{Xs}$ of Condition B were of equal intensity to the two $\mathrm{Xs}$ of Condition C. In Fig. 5 the latency data are plotted, showing the shorter latency of Condition $A$ vs Condition B. Again it should be pointed out that Condition $C$ produced shorter latencies than $B$ despite the greater intensities of stimuli in Condition $B$. The $t$ test for correlated data was again used to test for amplitude differences under the different conditions for P2, the largest positive VEP component. The two-tailed criterion was used in all tests of statistical significance. For P2 a significant difference was observed between Conditions $A$ and $B$, showing a higher P2 under A (blanking overcome) than B (blanking) $(t=4.13$, $\mathrm{p}<.01$, df $=5$ ).

The t-test results for latency differences show that all of the possible comparisons are highly significant $(p<.01, \mathrm{df}=5)$ for the $\mathrm{P} 2$ components, i.e., for P2, A vs B, $t=15.67 ; A$ vs $C, t=8.73$; and $B$ vs $C$, $t=7.19$. This indicates that these components occurred significantly later in Condition $\mathrm{C}$ than in Condition $A$ and later under $B$ than under $A$. In fact, P2, for example, occurred $33.3 \mathrm{msec}$ later in $\mathrm{C}$ than in $A$ and 71.6 msec later in $B$ than in $A$.

Figure 6 shows the overprinted VEPs for one of our Ss (J.A.). The differences in latencies under the three conditions, as well as the variations in VEP waveform, are notable.

\section{DISCUSSION}

In this second experiment we find evidence for two different statements: (1) Sequential blanking can be overcome by increasing the intensity of the normally blanked stimuli; and (2) perceptual suppression takes place in the sequential blanking paradigm when the blanking stimuli are of greater intensity than the normally blanked stimuli, and this suppression is accompanied by an apparent disappearance of the VEP response for the first of the two blanked stimuli. The evidence for these statements stems from analyses of the latencies of the major component of the VEP, i.e., P2. It was found that the latency of the P2 component was delayed by $71.6 \mathrm{msec}$ under Condition B (blanking strengthened) as compared to A (blanking overcome). There appear to be two factors operating to produce the longer latency of P2 under Condition $\mathbf{B}$ as compared to $\mathbf{A}$. The first factor might be the intensity difference between the first $X$ in Conditions A (.07 $\mathrm{mL})$ and $B$ $(.02 \mathrm{~mL})$. Intensity would appear to produce a latency difference of approximately $33.3 \mathrm{msec}$, a figure obtained by subtracting the P2 latency of Condition A (241.03 msec) from the P2 latency of Condition C $(274.35 \mathrm{msec})$. The second factor is one that might be labeled "suppression," which is most likely due to the action of the blanking stimuli upon the VEP response to the blanked Xs in Condition B. The VEP is delayed $38.3 \mathrm{msec}$ by this suppressing action, a figure obtained by subtracting the P2 latency of Condition $\mathrm{C}$ ( $274.35 \mathrm{msec}$ ) from that of Condition B (312.65 msec). This reasoning is based on the fact that the intensity of the first $\mathrm{X}$ in Condition $\mathrm{C}$ is the same as the first $X$ in Condition $B$, and, therefore, any difference in VEP latency between the two must have been caused by a factor other than intensity, i.e., the suppressing effect of the blanking stimuli.

The 38.3-msec delay between P2 in Conditions $\mathbf{B}$ and $\mathbf{C}$ is extremely close to the 40-msec delay which represents the time of appearance of the second $X$ in Condition $B$ and leads to the conclusion that there was no VEP response to the first $X$ (blanked) of Condition B, but rather that the VEP represents a response to the second $X$ (blanked) in that condition. The VEP may have occurred to the second $X$, even though it did not with the first, because of a stimulus summation effect on the neurons in the responding area of the visual cortex $\left(\mathrm{O}_{2}\right)$. That is to say, the suppression of response to the first $X$ prevented a VEP, but the additional stimulation provided by presentation of the second $X$ may have allowed response threshold to be reached for many of

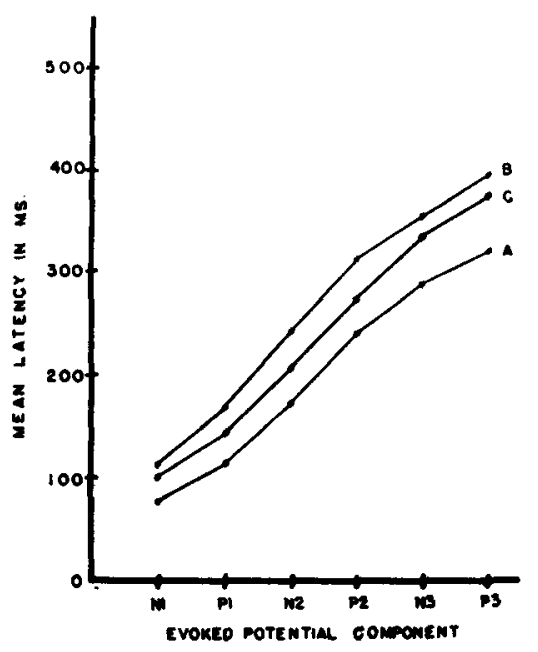

Fig. 5. Mean latencies (six Ss) for VEP components under Conditions $A$ (blanking overcome), B (blanking), and $C$ (two Xs).

the neurons in the responding area. Thus, in this second experiment there is some evidence for suppression of physiological response (VEP) to the first of the blanked stimuli, accompanied by a perceptual suppression to both of the blanked Xs. This result is similar to those of Donchin et al (1963) and Donchin and Lindsley (1965) since they report that the VEP did not occur in response to

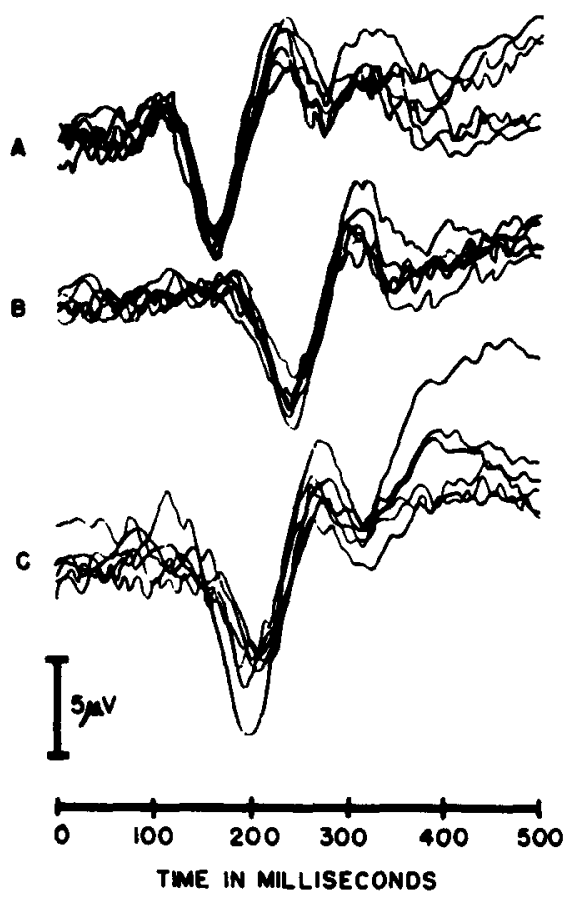

Fig. 6. VEPs of one S (J.A.) under Conditions A (blanking overcome), B (blanking), and C (two Xs). Negativity is downward. 
the earlier, weaker light flash in their visual masking experiments. The latency difference between $\mathrm{P} 2$ in Condition $\mathrm{C}$ as compared to $\mathrm{A}$ is consistent with findings of past investigators who reported longer VEP latencies with decreasing stimulus intensities (e.g., Vaughan \& Hull, 1965; Fuster \& Sierra, 1968).

In brief, it is concluded that under the normal conditions of sequential blanking (Experiment 1) in which all stimuli were of equal intensity, perceptual suppression occurred, but the VEP did not disappear. Under conditions in which the normally blanking stimuli were of greater intensity than the blanked stimuli (Experiment 2) not only did perceptual suppression occur, but physiological suppression was suggested by evidence that the VEP did not occur to the first of the two blanked stimuli. The differing results between Experiments 1 and 2 suggest the possibility that when the blanked and blanking stimuli are of equal intensity, the hypothesized interaction between the excitatory and inhibitory fields that occurs in sequential blanking paradigms [see Mayzner and Tresselt (1970) for a full discussion of excitatory-inhibitory interactions and their effects] assumes a different form than when the blanked and blanking stimuli are of unequal intensity. More specifically, it would appear from our findings that when the blanking stimuli are more intense (i.e., have a higher luminance) than the blanked stimuli, the action of the inhibitory fields of the blanking stimuli on the excitatory fields of the blanked stimuli is of sufficient magnitude to be reflected in the VEP responses (i.e., there is no response to the first of the two blanked $\mathrm{Xs}$ ), while, in contrast, when all stimuli are of equal intensity, the inhibitory action is correspondingly less and only a perceptual (subjective) response difference results.

\section{REFERENCES}

DONCHIN, E., \& COHEN, L. Averaged evoked potentials and in tramodality selective attention. Electroencephalography \& Clinical Neurophysiology, 1967, $22,537-546$.

DONCHIN, E., \& LINDSLEY, D. B. Visually evoked response correlates of perceptual masking and enhancement. Electroencephalography \& Clinical Neurophy siology, $1965,19,325-335$.

DONCHIN, E., WICKE, J., \& LINDSLEY, D. B. Cortical evoked potentials and perception of paired flashes. Science, $1963,141,1285-1286$.

EASON, R. G., HARTER, M. R., \& WHITE, C. T. Effects of attention and arousal on visually evoked potentials and reaction time in man. Physiology \& Behavior, $1969,4,283-289$

FUSTER, J. M., \& SIERRA, G. Amplitude of visual evoked potentials as a function of illuminance. Psychonomic Science, 1968, 12, 105-106.

GROVES, P. M., \& EASON, R. G. Effects of attention and activation on the visual evoked cortical potential and reaction time. Psychophysiology, 1969, 5 394-398.

HUBEL, D. H. Integrative processes in central visual pathways of the cat. Journal of the Optical Society of America, 1963, 53, 58-66.

JASPER, H. H. Report of the committee on methods of clinical examination in electroence phalography. Electroencephalography \& Clinical Neurophysiology, $1958,10,370-375$.

MAYZNER, M. S. The research potential of a computer-based cathode ray tube display system. Behavior Research Methods \& Instrumentation, 1968, 1, 41-43.

MAYZNER, M. S., \& TRESSELT, M. E. Visual information processing with sequential inputs: A general model for sequential blanking, displacement and overprinting phenomena. Annals of the New York Academy of Sciences, 1970, 169, 599-618.

MAYZNER, M. S., TRESSELT, M. E., \& HELFER, M. S. A provisional model of visual information processing with sequential inputs. Psychonomic Monograph Supplements, 1967, 2, 91-108.

MORGAN, C. T. Physiological psychology. (3rd ed.) New York: McGraw-Hill, 1965. SCHILLER, P. H., \& CHOROVER, S. L. Metacontrast: Its relation to evoked potentials. Science, 1966, 153. 1398-1400.

VAUGHAN, H. G., JR., \& HULL, R. C. Functional relation between stimulus intensity and photically evoked cerebral responses in man. Nature, 1965, 206, 720-722.

VAUGHAN, H. G., JR., \& SILVERSTEIN, L. Metacontrast and evoked potentials: A reappraisal. Science, 1968, 160, 207-208.

\section{NOTE}

1. Thus, under the fixation instructions of the study, designed to match those instructions employed in previous sequential blanking studies, it may be assumed that for most, if not all display presentations the display sequence occurred foveally, since most estimates of foveal area are about 2 deg of visual angle.

(Accepted for publication February 15, 1971.) 